\title{
Highly repeated DNA sequences in three species of the genus Pteropus (Megachiroptera, Mammalia)
}

\author{
MJL Barragán ${ }^{1}$, S Martínez ${ }^{1}$, JA Marchal ${ }^{1}$, M Bullejos ${ }^{1}$, R Díaz de la Guardia ${ }^{2}$ and \\ A Sánchez Bullejos ${ }^{1}$ \\ ${ }^{1}$ Departamento de Biología Experimental, Facultad de Ciencias Experimentales y de la Salud, Universidad de Jaén, E-23071 Jaén, \\ Spain; ${ }^{2}$ Departamento de Genética, Facultad de Ciencias, Universidad de Granada, E-18071 Granada, Spain
}

Bat genomes are characterised by an A-T richness and by
a small C-value compared with other mammalian groups. It
has been suggested that the small C-value is mainly due to
the lack of repetitive DNA sequences. However, little infor-
mation about repetitive DNA sequences in this mammalian
group is available at the molecular level. Here we describe
a Pstl family of repetitive DNA sequences present in three
species of the genus Pteropus. These repetitive sequences are $54.97 \%$ G-C rich, organised in tandem and with a unit length of $744 \mathrm{bp}$. Methylation analysis indicates that some of the CCGG target sites present in these repetitive DNA sequences have methylated cytosines and that there are small differences in the methylation pattern between species. Several features of this family of repetitive sequences suggest that they evolved by concerted evolution.

Heredity (2002) 88, 366-370. DOI: 10.1038/sj/hdy/6800064

Keywords: Pteropus; megachiroptera; repetitive DNA; satellite DNA

\section{Introduction}

Mammalian genomes are characterised by a large number of repetitive sequences, which are highly variable from one species to another. These sequences constitute families of repetitive DNA that represent a substantial component of many eukaryotic genomes. In fact, these types of sequences can represent values from less than $10 \%$ to more than $80 \%$ of the DNA content (Ridley, 1996). Two main kinds of highly repetitive sequences are known in mammalian genomes: interspersed DNA, in which the repeated DNA sequences are dispersed throughout the genome; and satellite DNA which is characterised by long tandem arrays and consistent association with constitutive heterochromatin (Singer, 1982). The localised repeats, or satellite DNAs, are typically found at C-band-positive regions of chromosomes, such as centromeres, telomeres and a portion of the $\mathrm{Y}$ chromosomes (Modi et al, 1988; Hamilton et al, 1990). The satellite DNAs, which are generally A-T rich, are organised in tandem arrays of repeat units and are highly variable with respect to the nucleotide sequence, the size of units, number of repeated units, and genome and chromosome organisation and localisation (Charlesworth et al, 1994).

The biological function of these DNA sequences remains elusive, although it has been proposed that they are involved in centromeric condensation, sister chromatid pairing, chromosome association with the mitotic spindle, karyotypic evolution and chromosome arrangement (Miklos and John, 1979; Singer, 1982; Vogt, 1992).

Correspondence: Dr A Sánchez Baca, Departamento de Biología Experimental, Facultad de Ciencias Experimentales, Universidad de Jaén, Paraje Las Lagunillas s/n, E-23071 Jaén, Spain. E-mail: abaca@ujaen.es Received 12 June 2001; accepted 3 January 2002
Bats possess a small $\mathrm{C}$ value (genome size is approximately $50-87 \%$ the genome size of other eutherian mammals: Burton et al, 1989) possibly due to control of copy number of repetitive DNA sequences. This decrease in DNA content is accompanied by a high overall A-T content (Pettigrew, 1994). Van Den Bussche et al (1995) analysed the frequency of three types of repeat sequences (dinucleotide microsatellites, ribosomal DNA cistrons and a family of repetitive DNA sequences) in the bat Macrotus waterhousii demonstrating that there is a lower copy number of interspersed and tandemly repetitive elements. These authors suggest that the difference in the nuclear content of bats in relation to other mammalian groups is mainly due to the lack of interspersed repetitive DNA sequences. Although repetitive DNA sequences have been studied in almost all mammalian groups, in general little information is available at the molecular level for these sequences in bats, the second largest mammalian order, which includes nearly 1000 species in existence.

In this study we present the characterisation of a PstI family of repetitive DNA sequences present in the genome of three bat species from the genus Pteropus.

\section{Materials and methods}

\section{Species sampled and DNA extraction}

Individuals of three species from the genus Pteropus were analysed. These species are $P$. scapulatus, $P$. alecto and $P$. poliocephalus. Genomic DNA was extracted from ethanolfixed tissues according to standard phenol-chloroform procedures (Sambrook et al, 1989).

Cloning and sequencing

In order to detect the repetitive DNA sequences genomic DNAs from Pteropus species were digested with NheI, 
PstI, EcoRI and AluI restriction endonuclesases. Only when genomic DNAs were digested with PstI was a prominent band of repetitive DNA found. PstI bands from the three species analysed were eluted from agarose and ligated to PstI digested pUC19 vector.

Escherichia coli JM109 competent cells were transformed with the ligation reactions and the recombinant bacteria containing the sequences of interest were selected after screening, using as a probe the band from $P$. alecto, digoxigenin-labelled by random priming (Roche).

The positive clones were sequenced in both directions using the Thermosequenase fluorescent cycle sequencing kit from Amersham. The sequence reactions were analysed on a $6.5 \%$ polyacrylamide gel in a LICOR-400L automated sequencer.

\section{Southern blot}

Genomic DNAs were digested with the appropriate restriction endonucleases. The fragments were separated in a $1 \%$ agarose gel and blotted onto nylon membranes (Amersham) according to Sambrook et al (1989). Membranes were probed with the P. alecto eluted band or with the clone P.ale 2.2 at $55^{\circ} \mathrm{C}$ overnight. Alkaline phosphatase detection was carried out according to the supplier's recommendations (Roche).

\section{Sequence analysis}

Pairwise sequence alignment and multiple alignment were carried out with the program CLUSTAL W 1.6 (Thompson et al, 1994). Genetic distances were calculated according to Kimura's (1980) two-parameter method, and the resulting distance matrices were subjected to neighbour-joining analysis (Saitou and Nei, 1987). A consensus tree was constructed, and the significance of the phylogenetic lineages was assessed by bootstrap analysis with 1000 replications. The phylogenetic analyses were carried out using the program MEGA Version 1.02 (Kumar et al, 1993).

\section{Results and discussion}

Digestion of genomic DNA from P. scapulatus, P. alecto and $P$. poliocephalus with PstI yielded a prominent band at about $744 \mathrm{bp}$ (Figure 1a). When genomic DNA of these three species was digested with PstI and probed with the digoxigenin-labelled $744 \mathrm{bp}$ band from $P$. alecto, a regular ladder pattern was seen (Figure $1 b$ ). In general, the size of each band corresponds to the size of multimers of the monomer unit of $744 \mathrm{bp}$. Nevertheless, some bands do not correspond to monomer multimers. In $P$. scapulatus and $P$. polocephalus there are also bands of $372 \mathrm{bp}$ and 186 $\mathrm{bp}$, which could be due to the presence in some monomer units of target sites for PstI. In fact several incomplete target sites for this enzyme are present in the sequences of some monomers. These sites need only one base substitution change to give rise to the complete target site.

The presence of a ladder pattern in Southern blots probed with a digoxigening-labelled 744 bp band clearly indicates that the repeated sequences in Pteropus are arrayed in tandem. It is likely, as occurs with other repeated DNA sequences present in eukaryotic genomes, that the bands containing several monomer units are originated by one base substitution change that modifies the target sequences for PstI.

When genomic DNA from other bat species, including

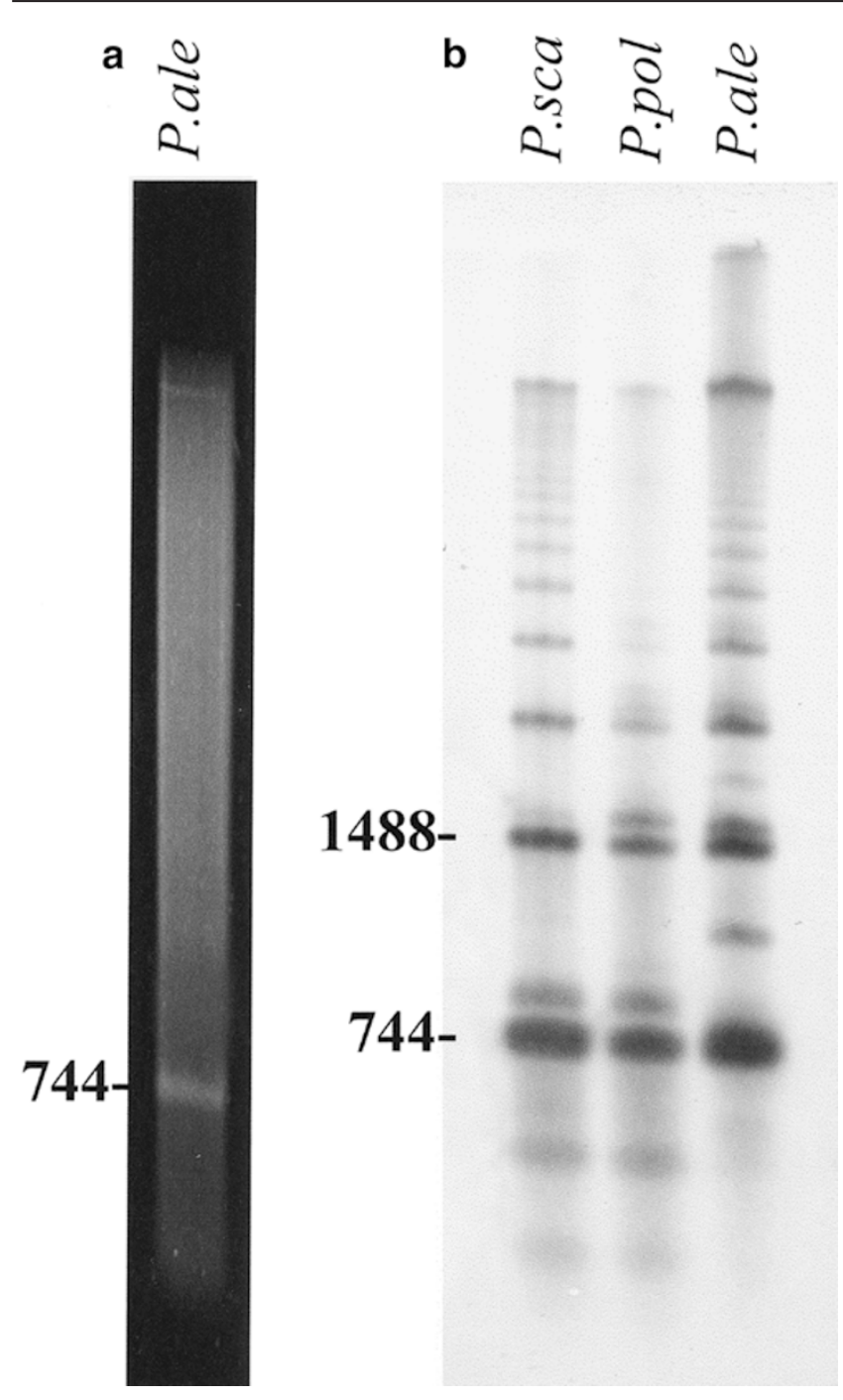

Figure 1 (a) Gel electrophoresis of PstI digested genomic DNA from Pteropus alecto. (b) Southern blot of PstI digested genomic DNAs from P. scapulatus (P.sca), P. poliocephalus (P.pol) and P. alecto (P.ale) probed with the random primer digoxigening-labelled 744 bp band of $P$. alecto.

a megabat (Rousettus aegyptiacus) and three microbats (Myotis myotis, Rhinolophus ferrumequinum and $R$. hipposideros), were digested with PstI and probed with the repetitive sequence of Pteropus, no band was observed (data not shown). This result implies that these sequences are absent in these micro- and megabat species and hence could be exclusive to the genus Pteropus.

After ligation with PstI digested pUC19 vector, transformation of E. coli and screening, positive clones were obtained from all the species of the genus Pteropus analysed: five positive clones from P. scapulatus: P.sca 19, 21, 27, 36 and 50; one from P. poliocephalus: P.pol 66 and two from P. alecto: P.ale 4.15 and 2.2 (with two monomer units, P.ale 2.2a and b).

Sequences from different clones showed that the satellite DNA cloned in Pteropus species belong to the same repetitive family. Sequence alignment allowed the determination of the consensus sequence for the monomer units. The length of the consensus sequence was $744 \mathrm{bp}$, although the length of cloned sequences ranged between 


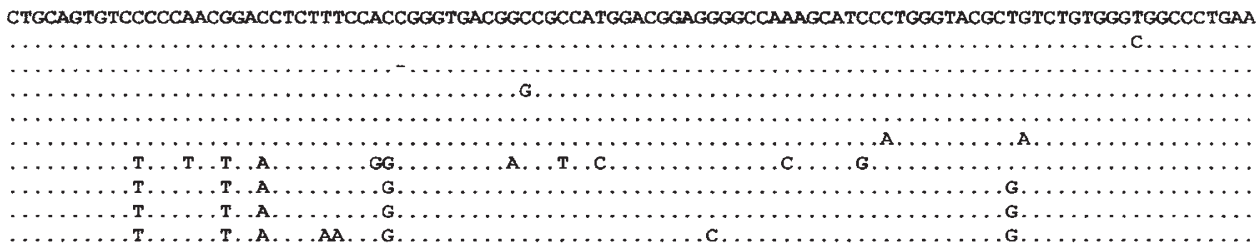

CCCTGCAAGAGGATACGGAGACGATTGAGACGTAGTTTGGACCTCTAGGAGCTCAAAGTGTCAGTGAGTGCTTCCCCAACTTCGGTACCCCAGTACCCGC

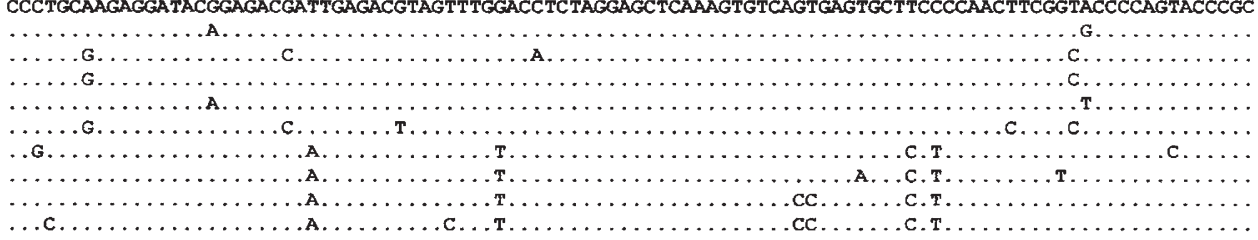

AGATGACCGAGCATCGCCCCCAGAGCCCCAAGGCGCCGGGAGGGCTGTGCATTTGCCGCAAGTCAGCCGTTTCTCGGAAGGTGCGATTCTGGCTCGAAA

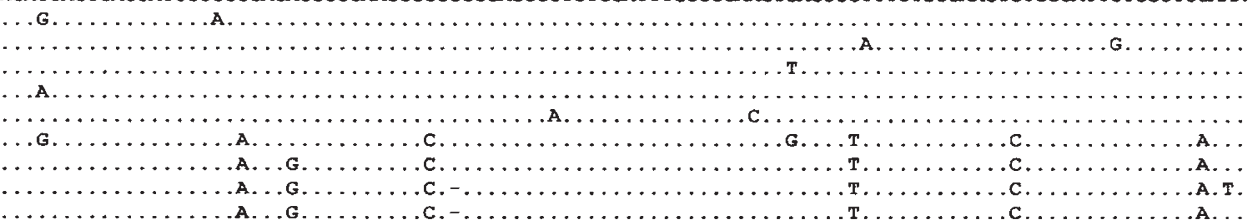

CGCACGTCACCAGAGAGCTTTTTGCCAAAGCTACCATATACATATATATGCATATATATGTTCATCTATATGCATATATATGTATATGGTGGATTTTCGA

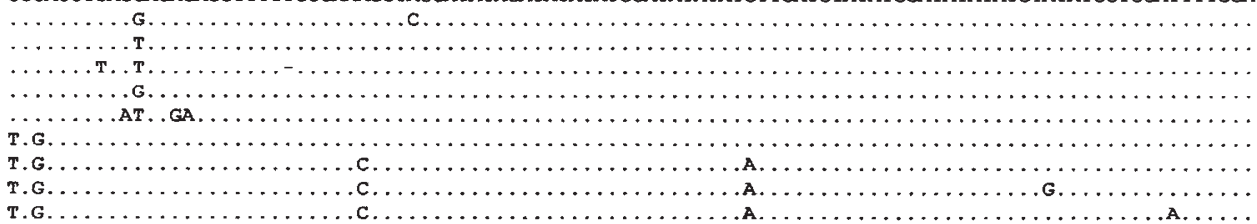

AGGAAACCTGTGTGGACCCCTGGGCTACCAGAGAAGACAGGCCGCTCCTAGCACCTCCACCTCCCCGCAGTGTGGACACCGGGCGTTCCAGAGCCACAAT
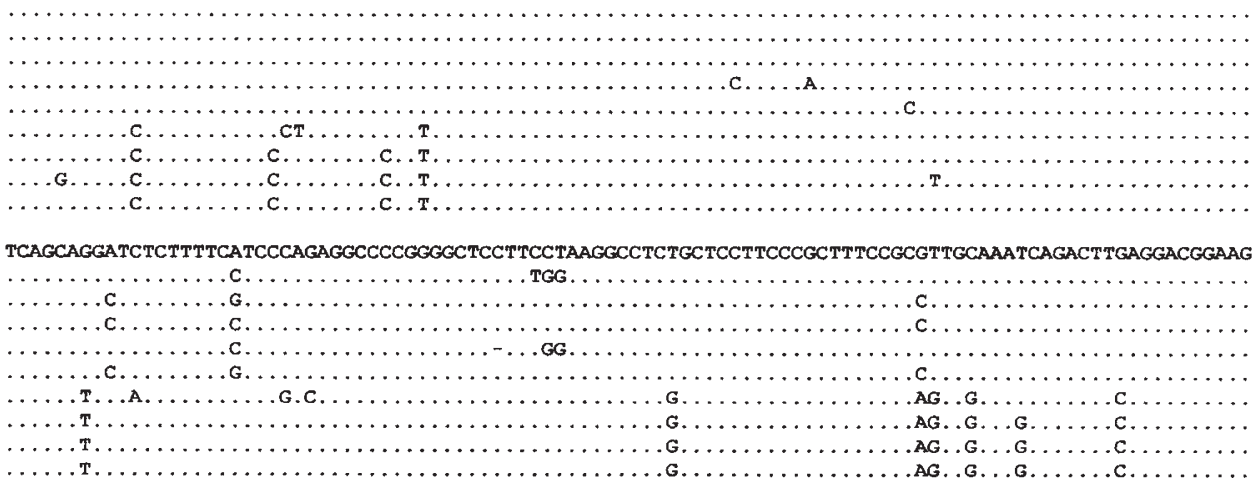

CAGACACCTCCCTCTCCCTCACCTTTCTGCCTGGGATGGTCGTAATGGTCCCCGTGGACGCATTCAAAATGAAGAAATGAAAGGCTCAGAGAGCTGATCG
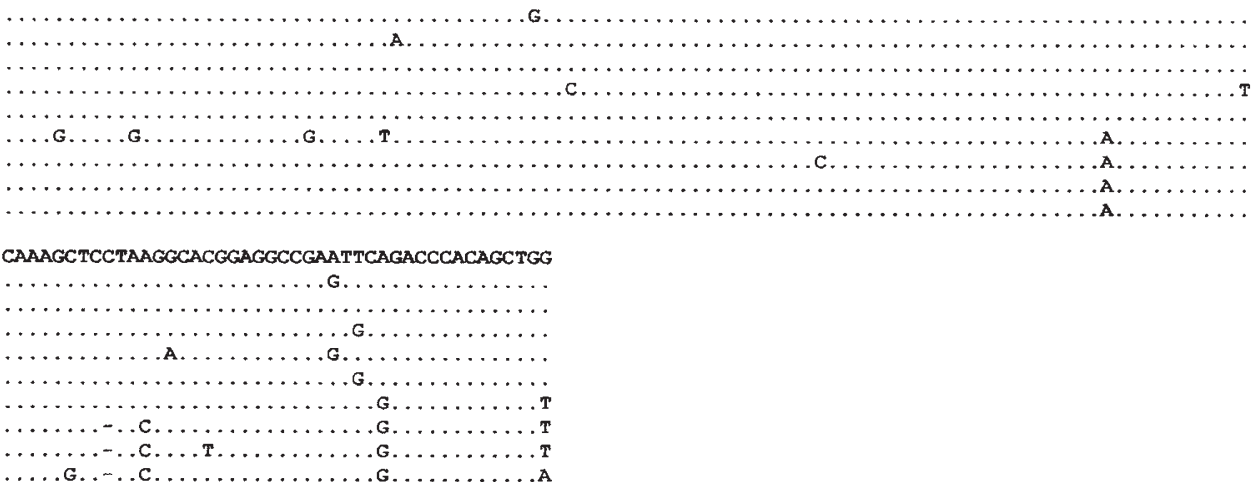

Figure 2 Sequence alignment of the consensus sequence with the different monomer units cloned from Pteropus scapulatus (P.sca), P. poliocephalus (P.pol) and P. alecto (P.ale). These sequences have been submitted to GenBank and have been assigned the accession numbers AJ311379 to AJ311387. 
744 and 742 bp (Figure 2). The consensus sequence is $54.97 \% \mathrm{G}-\mathrm{C}$ rich. A high A-T content is considered to be a general feature of most of satellite DNAs, as is the case for several rodent species which range from $60 \%$ to $63 \%$ (Singer, 1982). Furthermore, this higher G-C content for these sequences is interesting because the genomes of bat species are characterised by a high A-T content. In fact, the genomes of pteropodid bats have over $70 \%$ A-T content in their DNA (Petigrew and Kirsch, 1995).

Figure 2 shows the alignment of the consensus sequence with the cloned monomer units. Comparative analysis demonstrated that base substitution mutations are randomly spread along the sequences and that there is a high sequence identity, with the percentage between different monomer units ranging between 96 and $98 \%$. The same substitutions have occurred in several clones of this satellite DNA at the same position in the sequences. Such a mutational pattern could be explained as the result of homogenisation processes: by unequal crossing-over and amplification some particular monomer variations can be spread throughout the whole satellite (Dover, 1986). The evolution of satellite DNA is mainly determined by processes of concerted evolution. The concept 'concerted evolution' describes processes by which a sequence can be amplified, homogenised throughout the genome and distributed among both homologous and non-homologous chromosomes (Dover, 1982). Several mechanisms have been proposed to be active in certain genomes, such as unequal crossing over, gene conversion, sequence transposition and rolling circle replication (Dover, 1982; Walsh, 1987), which can act together or individually during the process of concerted evolution.

The consensus sequence from $P$. scapulatus monomers shows percentages of identity of $94.89 \%$ with the consensus sequence from $P$. alecto and of $93.41 \%$ with the single monomer unit from $P$. poliocephalus. The identity between $P$. alecto consensus sequence and $P$. poliocephalus monomer unit is $96.10 \%$.

As repetitive DNA sequences are known to be subject to hypermethylation, we investigated the methylation status in the CCGG sequences of this repetitive DNA in $P$. scapulatus and P. poliocephalus. The genomic DNA was digested independently with the methylation-insensitive enzyme MspI and with the methylation-sensitive enzyme HpaII. After Southern blot the membrane was probed with the clone P.ale 2.2 labelled with digoxigenin. In both species there are clear differences between the hybridisation pattern obtained with the two enzymes. While with MspI only two small bands showed hybridisation signal, with HpaII a typical ladder band was observed. These results are consistent with the fact that these repetitive DNA sequences have three or four target sites for these restriction endonucleases. Although the ladder obtained with HpaII in both species has the same main pattern, in $P$. scapulatus there are more interstitial bands than in $P$. poliocephalus (Figure 3). This result indicates that some of the CCGG target sites present in the repetitive DNA sequence have methylated cytosines in both species, and that there are small differences in the methylation pattern between them.

A neighbour-joining tree using the Kimura-2 parameter distance was constructed using nine monomer units from Pteropus species (Figure 4). Most branches are well supported including those that discriminate between

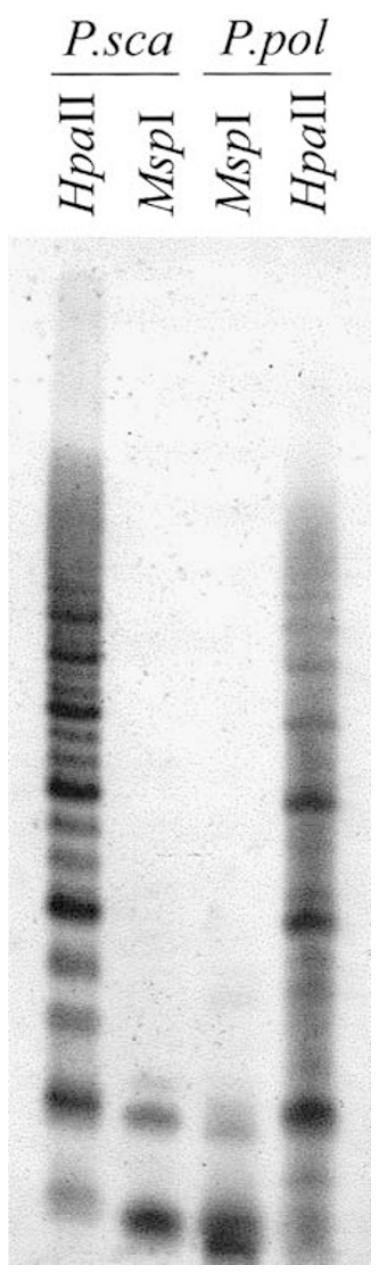

Figure 3 Southern blot of Pteropus scapulatus (P.sca) and P. poliocephalus (P.pol) genomic DNA digested with the methylation-insensitive enzyme MspI and with the methylation-sensitive enzyme HpaII, probed with the digoxigening-labelled clone P. ale 2.2.

species monomers. All monomer units from P. scapulatus are clearly located on a branch separated from those belonging to $P$. poliocephalus and $P$. alecto $(100 \%$ bootstrap value). Furthermore, the sequences for these two later species are also split into two separate branches with a bootstrap value of $99 \%$.

Phylogenetic inference reveals that genetic distances between repeat units of the same species were smaller than genetic distances between repeat units from different species. These data suggest a concerted mode of evolution for this satellite DNA family. Hence, the repetitive DNA sequences described here for the three Pteropus species can be considered as paralogous sequences. Those sequences could have arisen by duplication or amplification in the ancestral species and become slightly differentiated in the genome of each species. Concerted evolution has been demonstrated for other repetitive DNA from rodents, as in the case of the harvest mouse Reithrodontomys (Hamilton et al, 1990). In fact, the alignment of the monomer units from these species showed the existence of multiple diagnostic positions, that is, species-specific changes (Figure 2).

In conclusion, we described a Pst family of repetitive DNA sequences present in three megabat species belong- 

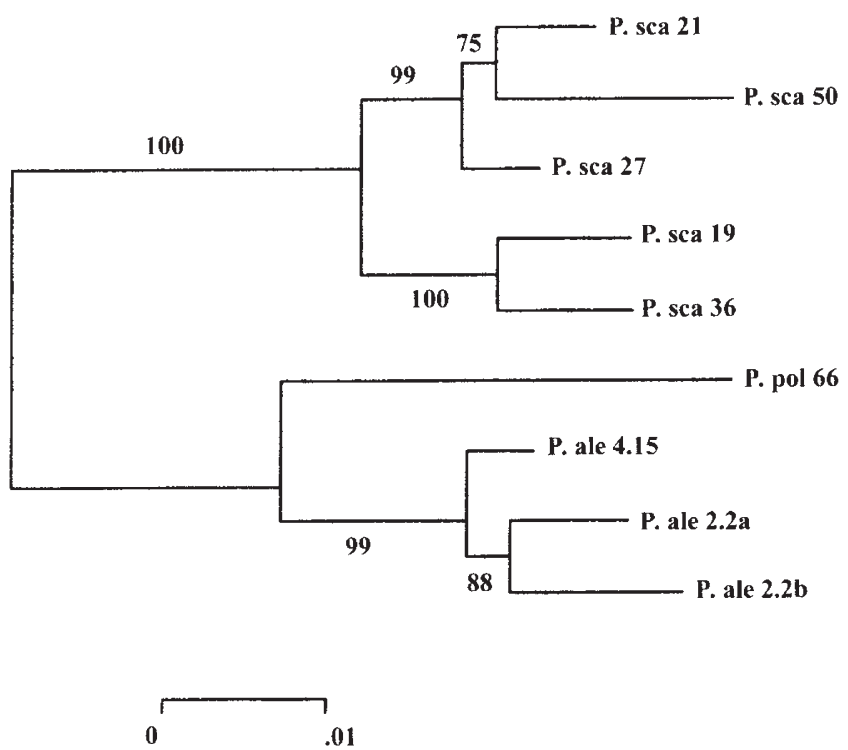

Figure 4 Neighbour-joining dendrogram showing the nine repetitive units of PstI satellite DNA from Pteropus species. The Kimura2 parameter distance was used for tree construction. P. sca \#, P. pol \# and P. ale \# are the different monomer units from P. scapulatus, $P$. poliocephalus and $P$. alecto respectively. The number in each branch indicates the bootstrap values (based on 1000 replicates). The branch length is drawn to the scale of the genetic distances (shown at bottom of figure). Note that monomers of each species are grouped in separate branches.

ing to the genus Pteropus. These repetitive sequences are organised in tandem arrays with a monomer unit of 744 bp and are $54.97 \%$ G-C rich. These sequences are clearly methylated in cytosine residues of the sequence CCGG. Furthermore, CLUSTAL alignment and phylogenetic analysis suggest that this family evolved by concerted evolution.

\section{Acknowledgements}

We would like to thank Dr Len Martin of Queensland University for generously providing megabat samples. This work was supported by the Spanish DGESIC through project numbers: PB98-1378-C02-01 and PB981378-C02-02, and by the Junta de Andalucía through the programme 'Ayudas a grupos de investigación', group numbers: CVI 0109 and CVI 220.

\section{References}

Burton DW, Bickhan JW, Genoways HH (1989). Flow-cytometric analysis of nuclear DNA content in four families of Neotropical bats. Evolution 34: 756-765.

Charlesworth B, Sniegowski P, Stephan W (1994). The evolutionary dynamics of repetitive DNA in eukaryotes. Nature 371: 215-220.

Dover GA (1982). Molecular drive: a cohesive mode of species evolution. Nature 199: 111-117.

Dover GA (1986). Molecular drive in multigene families: how biological novelties arise, spread and are assimilated. Trends Genet 168: 159-165.

Hamilton MJ, Honeycutt RL, Baker RJ (1990). Intragenomic movement, sequence amplification and concerted evolution in satellite DNA in harvest mice (Reithrodontomys): evidence from in situ hybridization. Chromosoma 99: 321-329.

Kimura M (1980). A simple method for estimating evolutionary rates of base substitution through comparative studies of nucleotide sequences. J Mol Evol 16: 111-120.

Kumar S, Tamura K, Nei M (1993). MEGA: Molecular Evolutionary Genetics Analysis, Version 1.01. The Pennsylvania State University, University Park, PA 16802.

Miklos GL, John B (1979). Heterochromatin and satellite DNA in man: properties and prospects. Am J Hum Genet 31: 264-280.

Modi WS, Fanning TG, Wayne RK, O'Brien SJ (1988). Chromosomal localization of satellite DNA sequences among $22 \mathrm{spec}-$ ies of felids and canids (Carnivora). Cytogenet Cell Genet 48: 208-213.

Pettigrew JD (1994). Flying DNA. Curr Biol 4: 277-280.

Pettigrew JD, Kirsch JA (1995). Flying primates revisited: DNA hybridization with fractionated, GC-enriched DNA. S Afr J Sci 91: 477-482.

Ridley M (1996). Evolution. Blackwell Science Inc: Cambridge, Mass, USA.

Saitou N, Nei M (1987). The neighbor-joining method: A new method for reconstructing phylogenetic trees. Mol Biol Evol 4: 406-425.

Sambrook J, Fritsch EF, Maniatis T (1989). Molecular Cloning. A Laboratory Manual, 2nd edn. Cold Spring Harbor Laboratory: Cold Spring Harbor, NY, USA.

Singer ML (1982). Highly repeated sequences in mammalian genomes. Int Rev Cytol 76: 67-112.

Thompson JD, Higgins DG, Gibson TJ (1994). CLUSTAL W: improving the sensitivity of progressive multiple sequence alignment through sequence weighting, positions-specific gap penalties and weight matrix choice. Nucl Acids Res 22: 46734680 .

Van Den Bussche RA, Longmire JL, Baker RJ (1995). How bats achieve a small C-value: frequency of repetitive DNA in Macrotus. Mammal Genome 6: 521-525.

Vogt P (1992). Code domainsin tandem repetitive DNA sequence structures. Chromosoma 101: 585-589.

Walsh JB (1987). Persistence of tandem arrays: Implication for satellite and simple-sequence DNAs. Genetics 115: 553-567. 